\title{
Vigilancia Tecnológica para la Identificación de Innovaciones en Subproductos de la Curtición
}

\author{
José B. Padilla ${ }^{4 *}$, Jhon W. Zartha ${ }^{2}$, Verónica T. Álvarez ${ }^{3}$ y Gina L. Orozco ${ }^{2}$
}

(1) Universidad del Quindío, Facultad de Ciencias básicas y tecnología, Carrera 15 Calle 12 Norte, ArmeniaColombia. (e-mail: jbpadilla@uniquindio.edu.co)

(2) Universidad Pontificia Bolivariana, Facultad de Ingeniería Agroindustrial, Circular 1ํㅡ \# 70-01 Laureles, Medellín-Colombia. (E-mail: jhon.zartha@upb.edu.co; gina.orozco@upb.edu.co)

(3) Universidad del Quindío, Facultad de Ciencias Agroindustriales, Carrera 15 Calle 12 Norte. Armenia-

Colombia. (e-mail: vtalvarezr@uqvirtual.edu.co)

${ }^{*}$ Autor a quien debe ser enviada la correspondencia

Recibido Nov. 20, 2017; Aceptado Feb. 5, 2018; Versión final Mar. 1, 2018, Publicado Ago. 2018

\section{Resumen}

El objetivo del trabajo que se presenta es la identificación de innovaciones y nuevos procesos en carnaza como subproducto de la curtición. Para esto se realizó un proceso de vigilancia tecnológica a través de una búsqueda de información, referente a juguetes caninos, tecnologías de proceso, normatividad, y tendencias. Se aplicó la metodología de análisis del ciclo de vida de la tecnología a través de curvas en S. Entre los documentos más importantes se encontraron dos nuevos procesos de elaboración para la fabricación de juguetes caninos descritos en dos patentes de Estados Unidos. Además, para la harina de carnaza su punto de inflexión en artículos se presentó en el periodo 2016-2018 lo cual representa una oportunidad para continuar investigando sobre esta tecnología.

Palabras clave: vigilancia tecnológica; carnaza; harina de carnaza; curtición; curvas $S$

\section{Technological Surveillance for the Identification of Innovations in Leather tanning byproducts}

\begin{abstract}
:
The objective of the work presented in this paper is the identification of innovations and new processes in split leather. For this, a process of technological surveillance was done through the search of information referred to canine toys, process technologies, normativity, and trends. Life cycle analysis of the technologies through S curves was applied. Among the most important documents found were two new processes for the manufacture of canine toys described in two patents in the United States. Also, for split leather flour its inflection point in articles was presented in the period 2016-2018, which represents an opportunity to continue research on this technology.
\end{abstract}




\section{INTRODUCCIÓN}

La carnaza es un subproducto que se convierte en un desecho industrial dentro del campo de las curtiembres, constituido por residuos de carne y sebo que acompañan a las pieles de los bovinos y se separan de ellas en el proceso de precurtición del cuero del animal. Esa capa del lado de la carne del cuero o de la piel animal, se puede usar como cuero pero tiene menor resistencia y menor valor comercial, este cuero también puede ser utilizado como guantes en la captura y/o manejo de aves debido a su resistencia (Freeman, Lemen, 2009), además tiene otros usos como materia prima para gelatina y se puede extraer el colágeno $100 \%$ puro usado en la industria farmacéutica, cosmética y de alimentos. (Vargas y Zuleta, 2010), por último, este tipo de cuero de baja calidad puede ser combinado con poliuretano para crear estructuras más resistentes y rígidas (Milašienè, 2007). Agroindustrias como las curtiembres han sido por mucho tiempo motivo de preocupación para organizaciones que se dedican a preservar el medio ambiente, por los residuos generados en dicho proceso. (Cámara de Comercio de Bogotá, 2004). Esta actividad industrial es, además, altamente demandante de recursos naturales, por cada $1000 \mathrm{~kg}$ de piel salada que entran al proceso, se requieren de cantidades considerables de agua e insumos químicos. (Centro Nacional de Producción Más Limpia, 2004).

Cualquier sector industrial, incluyendo los subsectores agroindustriales no alimentarios como el de la curtición necesitan del análisis, difusión y explotación de información técnica útil para la permanencia y crecimiento, por lo que son susceptibles de ser analizados a través de estudios Vigilancia Tecnológica (VT) la cual se define como un proceso organizado, selectivo y permanente, de captar información del exterior y de la propia organización sobre ciencia y tecnología, seleccionarla, analizarla, difundirla y comunicarla, para convertirla en conocimiento, para tomar decisiones con menor riesgo y poder anticiparse a los cambios. (UNE 166006:2011), otra de las ventajas de la vigilancia tecnológica está relacionada con la facilidad en la detección de las tecnologías e innovaciones emergentes y de la evolución de las tecnologías que suscitan especial interés en un campo especifico seleccionado. (Manderieux, 2011).

Una de las metodologías que agregan valor a la VT es el análisis de las Curvas en para identificar el estado de desarrollo de una tecnología. En los estudios relacionados al uso de las Curvas en $\mathrm{S}$ se identifican diferentes etapas o fases delimitadas por puntos específicos en su crecimiento tal como se muestra en la figura 1. Esta evolución ha sido estudiada por autores como Nelson y Winter (1997), Dosi (1982), Pérez (2001), Ortiz y Pedroza, (2006), Kucharavy y De Guio (2007), entre otros, también se encuentran nuevos estudios donde se analizan la cantidad de artículos en función del tiempo y se identifican periodos exploratorios, de desarrollos rápidos y de maduración. (Li, Chen y Kou, 2017). La metodología de Curvas e $S$ comprende varias fases las cuales tienen en cuenta trabajos previos en cuanto a nivel cualitativo (Burgelman, 2009; Schilling, 2013; Tidd y Bessant, 2013), y a nivel cuantitativo (Schilling, 2009; Foster, 1986), esta fases resumen los pasos a tener en cuenta para el cálculo del punto de inflexión partiendo de parámetros de desempeño como artículos y patentes acumuladas en el tiempo a través de técnicas de regresión no lineal.

Éste paper presenta los resultados del proyecto "usos y aplicaciones de la carnaza en diferentes sectores, priorizando el alimentario" ejecutado por la Universidad del Quindío - Colombia, el cual tiene como uno de sus objetivos impactar a las empresas del sector de Curtición a través de la identificación de nuevos procesos relacionados con la fabricación de juguetes caninos e identificar innovaciones de productos obtenidos a partir de la carnaza como subproducto de la curtición, la metodología utilizada fue la de Vigilancia Tecnológica desarrollada por Sánchez y Palop (2002) mediante la búsqueda de información en bases de datos especializadas en artículos y patentes referentes a carnaza, además de la aplicación de la metodología de análisis del ciclo de vida de la tecnología - curvas en S en una de las tecnologías elegidas, en este caso, la tecnología que se utilizó para dicho análisis fue "Harina de Carnaza".

\section{MATERIALES Y METODOS}

La metodología que se trabajó en este artículo fue la de vigilancia tecnológica y el análisis de ciclo de vida de la tecnología - curvas en S, a continuación, se observan los pasos de cada una de estas:

\section{Vigilancia Tecnológica}

De acuerdo a los factores críticos de vigilancia, se realizó la planeación, búsqueda y análisis de información alrededor del tema de interés, con el fin de identificar innovaciones, nuevos procesos y temas de valor agregado al proyecto, junto con el análisis de curvas en S. Es importante resaltar que la construcción de esta información necesitó de la participación de los investigadores de la Universidad del Quindío, y del coordinador general de curtiembre de la maría, quienes conocen el direccionamiento del estudio y su aplicación, quienes construyeron la información relacionada con Temas, Subtemas, Descriptores, 
Condicionantes y Nivel de Prioridad de la información, esto con el fin de tener toda la información disponible del proyecto para el estudio.

Los factores críticos de vigilancia se obtuvieron gracias al diligenciamiento por parte de José Bestier Padilla Docente Investigador de la Universidad del Quindío y José Joaquín Duque Coordinador general de la asociación de curtidores de la María. Además de los factores críticos, se definieron las necesidades del estudio, todos estos aspectos fueron claves para la realización del estudio de Vigilancia Tecnológica y Curvas en $\mathrm{S}$. Después de recibir los factores críticos de vigilancia, se identificaron las siguientes palabras claves, para la construcción de las ecuaciones de búsqueda: juguetes caninos, normas sanitarias, tecnologías metodologías de fabricación y subproductos de la carnaza.

De acuerdo con Sánchez y Palop, (2002), definen la metodología a seguir, la cual consiste en realizar inicialmente una planeación previa con los investigadores, con el fin de determinar los temas a vigilar mediante los factores críticos de vigilancia, después se procede a construir las ecuaciones de búsqueda, para artículos y patentes, estas son fundamentales en la captación de información en bases de datos especializadas, para este estudio se escogió la base de datos Scopus y Free Patents Online (FPO), debido a que son bases de gran impacto a nivel internacional. (Figura 2)

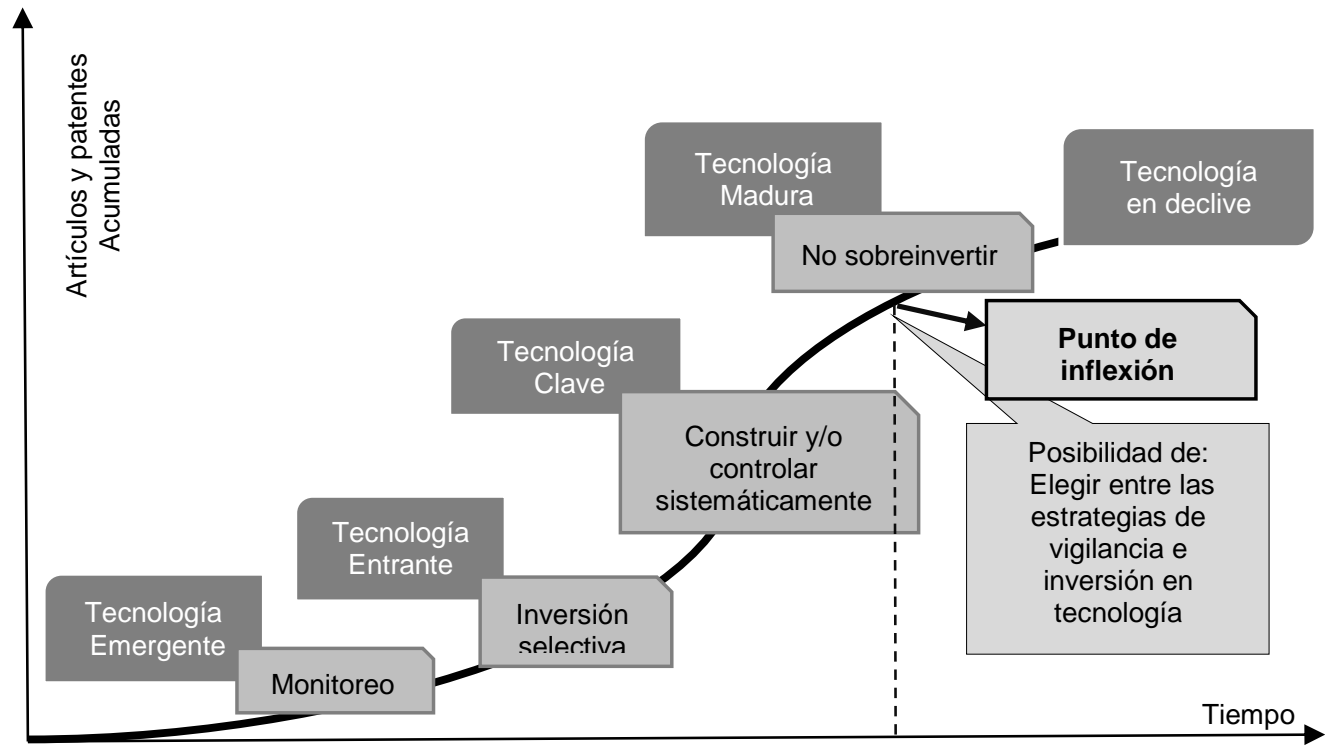

Fig. 1: Evolución de una tecnología (Modificado de Ortiz y Pedrosa, 2006)

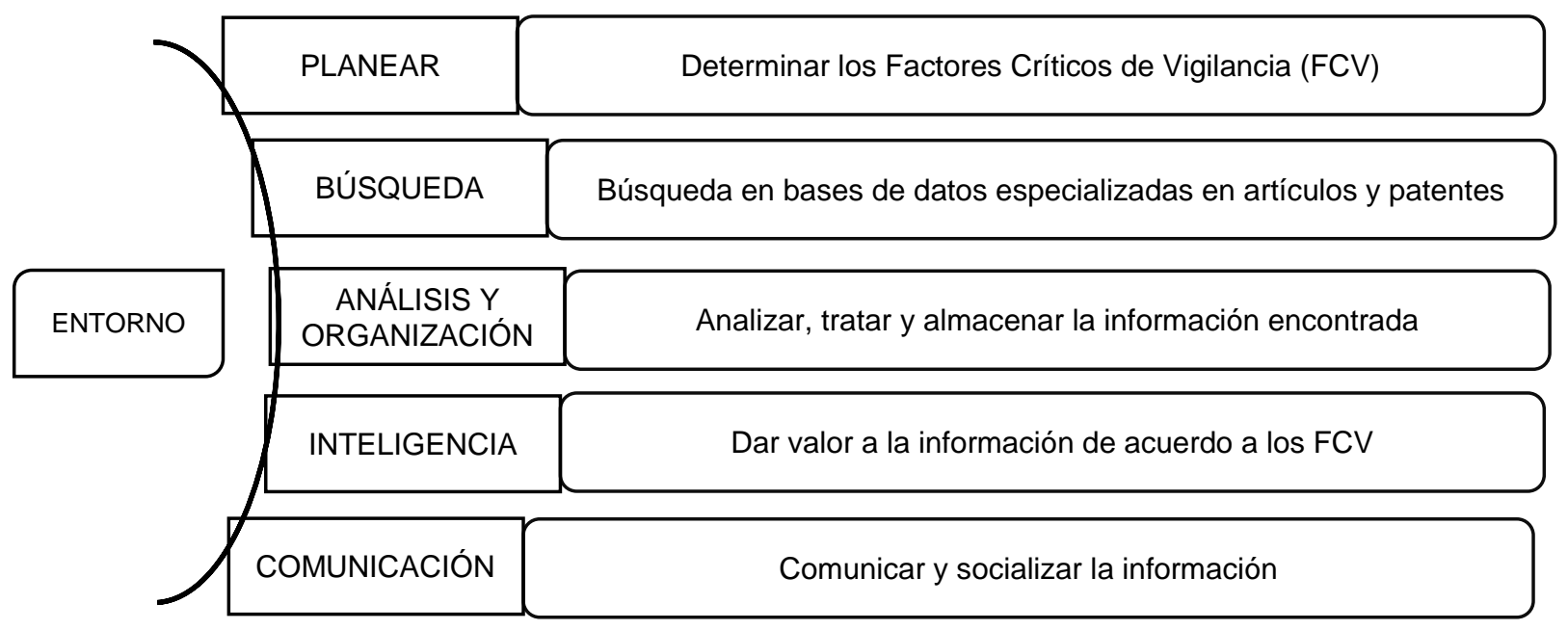

Fig. 2: Metodología VT (Adaptado de Sánchez y Palop, 2002)

\section{Análisis del ciclo de vida de la tecnología}

Las curvas en S permiten determinar el estado de la tecnología dentro de su ciclo de vida, de esta manera se disminuye la incertidumbre y toma de decisiones con un punto de referencia. Es por esto, que se 
selecciona una tecnología para dicho análisis, posterior a esto, se determina las ecuaciones de búsqueda para artículos y patentes y se acumula los datos para definir el parámetro de desempeño. Todo lo anterior como insumo para aplicar trece modelos de regresión no lineal.

El software utilizado fue Sigmaplot versión 13, bajo descarga gratuita, en este software se aplicaron a los datos acumulados de patentes y artículos los modelos de regresión no lineal: Sigmoidal 3 parámetros, Sigmoidal 4 parámetros, Sigmoidal 5 parámetros, Logístico 3 parámetros, Logístico 4 parámetros, Weibull 4 parámetros, Weibull 5 parámetros, Gompertz 3 parámetros, Gompertz 4 parámetros, Hill 3 parámetros, Hill 4 parámetros, Chapman 3 parámetros y Chapman 4 parámetros.

Aplicar los trece modelos tiene por objetivo encontrar el punto de inflexión, que es aquel que indica el momento en el que se encuentra la tecnología. Después de aplicar los trece modelos a la tecnología seleccionada en los datos de artículos y patentes, se selecciona el o los modelos que presentan mejor ajuste según los parámetros estadísticos establecidos, los cuales son: valor t: mayor que 2 y menor que -2; valor p: menor que 0.005; Durbin Watson: entre 1.8 y 2.2; $\mathrm{R}^{2}$ Ajustado: con valores cercanos a 1. Por último, se observa en la figura 3 , la metodología a seguir para las curvas en $\mathrm{S}$.

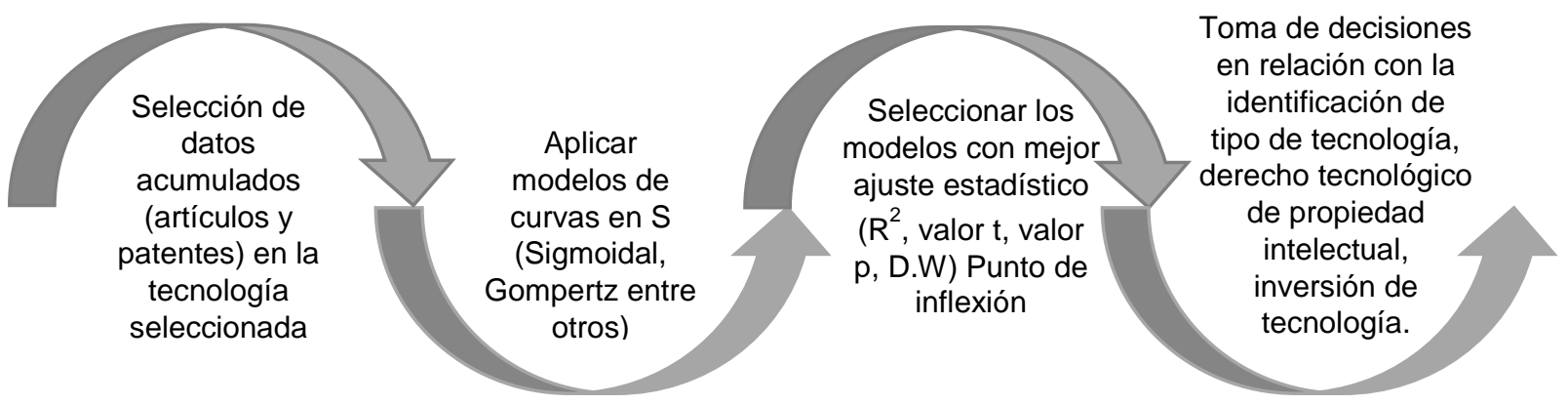

Fig. 3: Metodología curvas en S (Zartha et al. 2013)

\section{RESULTADOS}

Los resultados de éste estudio se clasifican en las siguientes fases:

Fase de planeación: los principales temas definidos en los factores críticos de vigilancia giraron en torno a juguetes para caninos, tecnologías de proceso, normatividad, tendencias, nuevas tecnologías (harina de carnaza), aplicaciones, nuevos productos, países e institutos pioneros en la investigación sobre carnaza, áreas temáticas de investigación, entre otros, estos aspectos fueron claves para la realización del estudio de vigilancia tecnológica y curvas en $\mathrm{S}$.

Fase de búsqueda: de acuerdo con los factores críticos de vigilancia se construyeron las ecuaciones de búsqueda, para proceder a identificar, clasificar, seleccionar y tratar la información obtenida de los artículos y patentes, con el fin de dar respuesta a los requerimientos descritos por los investigadores. Después de ser validada las palabras clave y la ecuación de búsqueda, se establecieron dichas ecuaciones. (Tabla 1)

Tabla 1: Ecuaciones de búsqueda validadas

\begin{tabular}{|c|c|c|}
\hline \multicolumn{3}{|l|}{ Artículos } \\
\hline Ecuación de búsqueda & $N^{\circ}$ de artículos & Base de Datos \\
\hline $\begin{array}{l}\text { TITLE-ABS-KEY("split leather" OR "carnaza") OR (process } \\
\text { OR "sanitary standard" OR technology AND "new products" } \\
\text { OR byproduct OR marketing AND applications OR uses AND } \\
\text { "manufacturing methodology") }\end{array}$ & 166 & Scopus \\
\hline \multicolumn{3}{|l|}{ Patentes } \\
\hline Ecuación de búsqueda & $\mathrm{N}^{\circ}$ de patentes & Base de Datos \\
\hline $\begin{array}{l}\text { ACLM/("split leather" OR "carnaza") AND (process OR } \\
\text { "sanitary standard" OR technology AND "new products" OR } \\
\text { byproduct OR marketing AND applications OR uses AND } \\
\text { "manufacturing methodology") }\end{array}$ & 142 & FPO \\
\hline
\end{tabular}


Después de haber sido validada las ecuaciones de búsqueda para artículos y patentes, en las bases de datos especializadas, se inició a extraer la información gráficamente, iniciando por artículos.

\section{Fase de análisis y organización:}

En esta parte se iniciará con los artículos científicos y luego la parte técnica que se evidencio en las patentes revisadas.

\section{Artículos}

Con base en los resultados obtenidos para artículos en la base de datos Scopus, a continuación, se muestra de forma gráfica los resultados obtenidos: en relación con la dinámica de publicación, se observa un incremento en el año 2002, y en los últimos cinco años se denota un creciente interés en el tema tratado. (Figura 4)

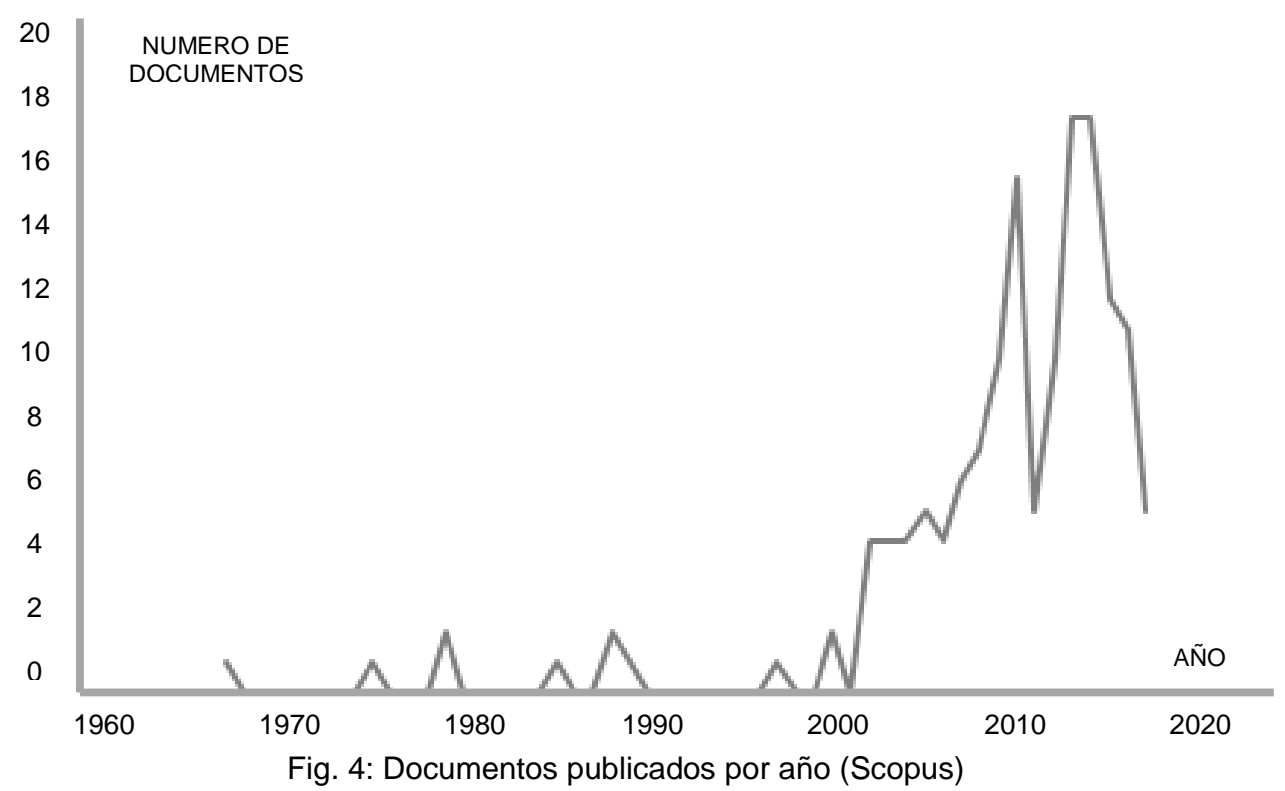

Las instituciones y Universidades con mayor publicación son "Pennsylvania State University" y "Hong Kong University of Science and Technology" con 6 documentos, "Chalmers University of Technology", "Universidade Federal do Rio Grande do Sul", "National Chiao Tung University Taiwan", "University of Windsor", "Georgia Institute of Technology", "Sultan Qaboos University", "The George W. Woodruff School of Mechanical Engineering" y "Siksha O Anusandhan University" con 4 documentos cada una. (Figura 5).

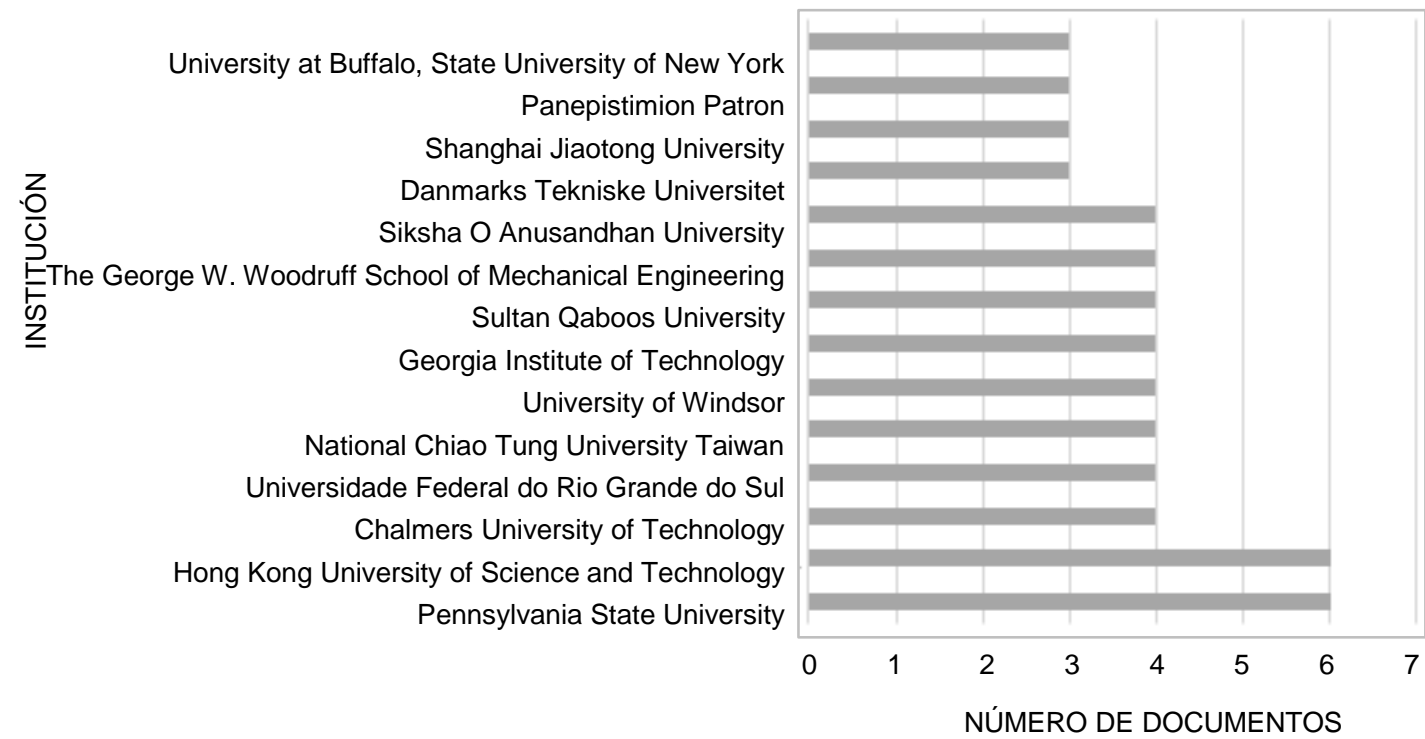

Fig. 5: Documentos por institución (Scopus) 
Los países con mayor publicación son Estados Unidos con 36 documentos, China con 21 documentos, United Kingdom con 18 documentos, Canadá con 12 documentos, Hong Kong y Taiwan con 10 documentos cada uno. (Figura 6)

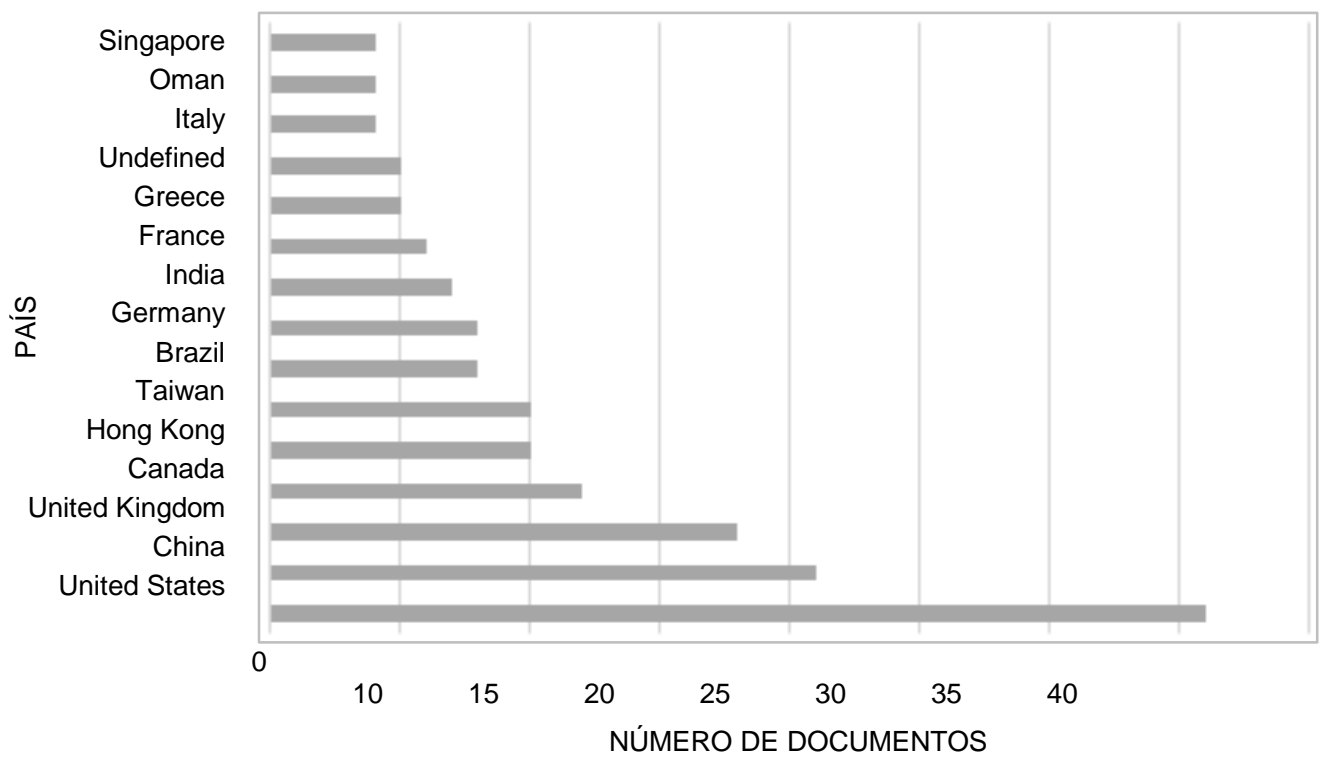

Fig. 6: Documentos por país (Scopus)

Por último, los documentos por área temática de mayor publicación son en Ingeniería, Ciencias de la Computación, Negocios, Gestión y Contabilidad, Ciencias de la Decisión, Matemáticas, Ciencia de los Materiales y Química, entre otros. (Figura 7)

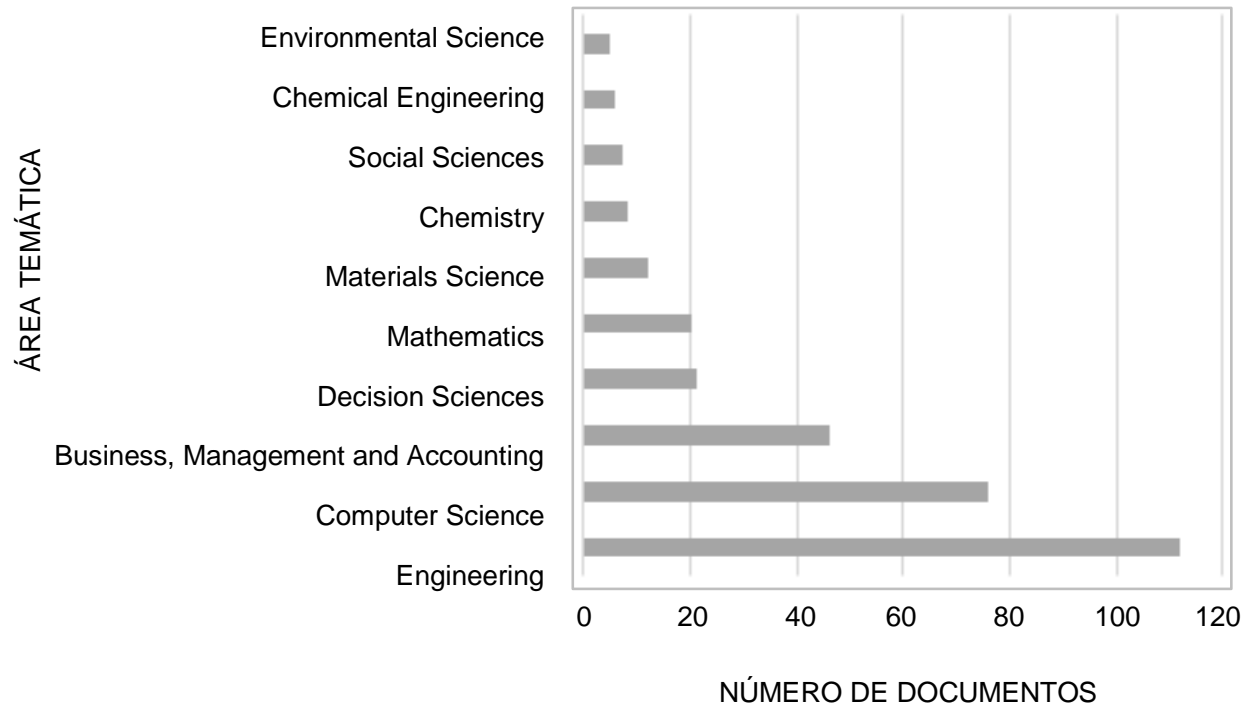

Fig. 7: Documentos por área temática

Por otra parte, para este estudio, se procedió a extraer la información gráficamente para patentes, con especial interés de conocer la base de datos más utilizada y la dinámica de solicitud de protección de invenciones.

\section{Patentes}

De acuerdo con los resultados obtenidos para patentes en la base de datos Free Patents Online (FPO), la oficina de patentes de Japón y Alemania son las más utilizadas para la solicitud de protección en el área de estudio. (Figura 8) 


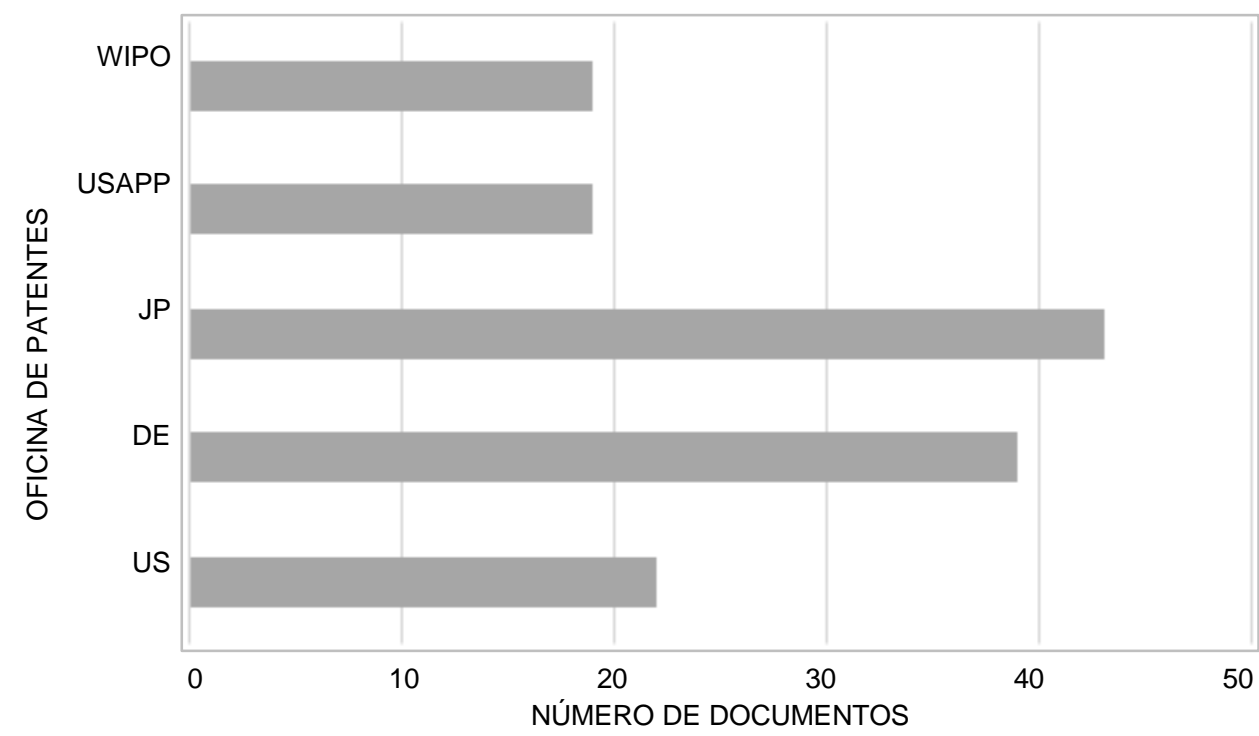

Fig. 8: Documentos en bases de datos (FPO)

En cuanto a las solicitudes de patente, se presentó un crecimiento entre los años 2006 al 2013, ahora viene en descenso, esto puede deberse a que las tecnologías y procedimientos en el sector no son tan dinámicos. (Figura 9)

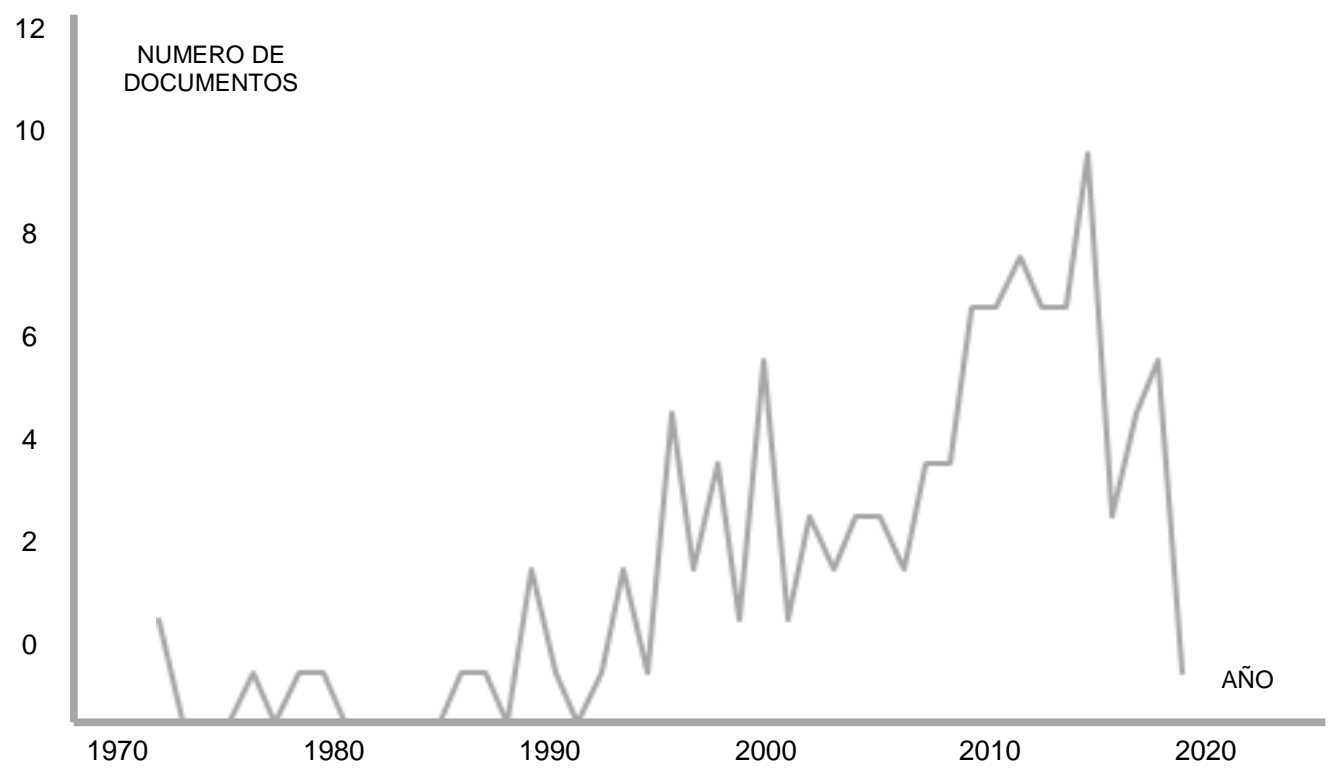

Fig. 9: Documentos por año (FPO)

\section{Fase de inteligencia:}

A continuación, se describe de forma esquemática los resultados de la vigilancia tecnológica en artículos y patentes, dando respuesta a los factores críticos de vigilancia, en temas como: juguetes para perros (nuevos procesos), procesos, productos y/o Nuevos Productos y/o Nuevas Aplicaciones (Alimentos, suplementos alimenticios, alimentos animales domésticos), usos, normatividad, tecnologías, productos químicos y vegetales y máquinas. (Tabla 2)

Tabla 2: Resultados vigilancia tecnológica

\begin{tabular}{|c|c|}
\hline Palabras Clave & Resultados \\
\hline $\begin{array}{lr}\text { Juguetes } & \text { para } \\
\text { perros } & \text { (nuevos } \\
\text { procesos) } & \end{array}$ & $\begin{array}{l}\text { Edible pet chew and preparation of same US20120079992A1 } \\
\text { Shanghai, China. Pet-Toys Industrial Co. } \\
\text { Artificial pet chewing leather, manufacturing method and application thereof US } 9320262 \text { B2 }\end{array}$ \\
\hline
\end{tabular}


Tabla 2 (continuación)

\begin{tabular}{|c|c|}
\hline Procesos & $\begin{array}{l}\text { Evaporación } \\
\text { Laminación } \\
\text { Calentamiento dieléctrico } \\
\text { Gofrado } \\
\text { Prensado térmico } \\
\text { Proceso de preparación, curtido, pre-teñido, teñido, secado, acabado, bronceado, punzonado. } \\
\text { Textiles tricotados multicapa } \\
\text { Revestimiento de fondo } \\
\text { Recurtido } \\
\text { Proceso de encalado } \\
\text { Emulsiones acuosas de poliuretano como agentes mejoradores para producir productos finales } \\
\text { que se comparan favorablemente con el grano natural. } \\
\text { Konrad et al. (2002) mencionan dos procesos de curtido ideales: el escenario } 1 \text { es un proceso } \\
\text { convencional de bronceado con cromo, y el escenario } 2 \text { es un proceso de curtido al cromo con } \\
\text { separación en el tanyard (etapa azul húmedo) y la tecnología de tambor aplicada en el haz de } \\
\text { luz. Con estos dos cambios, el consumo de agua y la generación de residuos sólidos podrían } \\
\text { reducirse significativamente. }\end{array}$ \\
\hline $\begin{array}{l}\text { Productos y/o } \\
\text { Nuevos Productos } \\
\text { y/o Nuevas } \\
\text { Aplicaciones } \\
\text { (Alimentos, } \\
\text { suplementos } \\
\text { alimenticios, } \\
\text { alimentos animales } \\
\text { domésticos) }\end{array}$ & $\begin{array}{l}\text { Láminas de poliuretano adheridos a cuero (Impermeabilidad). } \\
\text { Revestimiento de textiles con poliuretanos } \\
\text { Automotriz (Ej. cuero para asientos de automóviles, aviones, motos, etc) } \\
\text { Guantes industriales (Ej. guante de cuero siliconado); Cartuchera; Faltriquera (Ej. carriel) } \\
\text { Bolsas; Parte superior e inferior del zapato; Suela de zapatos (Ej. Zapatos de golf) } \\
\text { Cuero para cabrilla de automóviles; Guarnicionería (Ej. sillas de montar); Forros; Muebles; } \\
\text { Vestidos; Encuadernación (Ej. Libros) } \\
\text { Juguetes para aves (Ej. Tiras de cuero) } \\
\text { Bola de béisbol } \\
\text { La aplicación de una película de poliuretano micro porosa sobre cuero dividido por un proceso de } \\
\text { reacción conduce a cuero de primera calidad para la parte superior del calzado. (Schmelzer, 2016) } \\
\text { Colágeno (materia prima para gelatina). }\end{array}$ \\
\hline Usos & Aislamiento; Resistencia al agua; Propiedades químicas \\
\hline Normatividad & $\begin{array}{l}\text { Método de prueba estándar para la aceptación (ASTM “Standard Test Method for Acceptance”) } \\
\text { Sistema de Gestión de la Calidad (NTC ISO 9001) } \\
\text { Sistema de Gestión Ambiental (NTC ISO 14001) } \\
\text { Usos del Agua y Residuos Líquidos (Decreto } 1594 \text { de } 1984 \text { - Derogado) } \\
\text { Decreto } 321 \text { de } 1999 ; 4741 \text { de 2005; } 1609 \text { de } 2002 ; 3930 \text { de } 2010 . \\
\text { Resolución } 0631 \text { de 2015; } 909 \text { de } 2008 ; 1541 \text { de } 2013 .\end{array}$ \\
\hline Tecnologías & Saddler (Guarnicionería) Alemania \\
\hline $\begin{array}{l}\text { Productos químicos } \\
\text { y vegetales }\end{array}$ & Resinas de poliuretano termoendurecibles; Taninos vegetales; Taninos sintéticos \\
\hline Maquinas & Rodillo; Rodillo Inverso; Horno; Equipo de recubrimiento de cuchilla; Prensa \\
\hline
\end{tabular}

\section{Análisis del ciclo de vida de la tecnología - Curvas en S}

De acuerdo con la tecnología seleccionada para este análisis "Harina de Carnaza", se construyó las ecuaciones de búsqueda. (Tabla 3)

Tabla 3: Ecuaciones de búsqueda validadas para la tecnología "Harina de Carnaza"

\begin{tabular}{|c|c|c|}
\hline \multicolumn{3}{|l|}{ Artículos } \\
\hline Ecuación de búsqueda & $\mathrm{N}^{\circ}$ de artículos & Base de Datos \\
\hline $\begin{array}{l}\text { TITLE-ABS-KEY(technology flour AND "split leather" OR "tallow" OR } \\
\text { obtain* AND process) }\end{array}$ & 104 & Scopus \\
\hline \multicolumn{3}{|l|}{ Patentes } \\
\hline Ecuación de búsqueda & $\mathrm{N}^{\circ}$ de patentes & Base de Datos \\
\hline $\begin{array}{l}\text { ABST/("technology flour" AND "split leather" OR "tallow" AND } \\
\text { obtain* AND process) }\end{array}$ & 73 & FPO \\
\hline
\end{tabular}


Con los resultados de la aplicación para las ecuaciones de búsqueda en las bases de datos, se generó la serie de datos acumulados en artículos y patentes para ser ingresados al software Sigmaplot. (Tabla 4)

Tabla 4: Datos acumulados en Artículos y Patentes (Scopus y FPO)

\begin{tabular}{|c|c|c|c|c|c|}
\hline Año & Dato & Acumulado & Aก̃o & Dato & Acumulado \\
\hline \multicolumn{3}{|c|}{ Artículos } & \multicolumn{3}{|c|}{ Patentes } \\
\hline 1969 & 1 & 1 & & & \\
\hline 1970 & 0 & 1 & & & \\
\hline 1971 & 0 & 1 & 1971 & 1 & 1 \\
\hline 1972 & 0 & 1 & 1972 & 0 & 1 \\
\hline 1973 & 0 & 1 & 1973 & 2 & 3 \\
\hline 1974 & 0 & 1 & 1974 & 0 & 3 \\
\hline 1975 & 0 & 1 & 1975 & 0 & 3 \\
\hline 1976 & 0 & 1 & 1976 & 1 & 4 \\
\hline 1977 & 1 & 2 & 1977 & 0 & 4 \\
\hline 1978 & 0 & 2 & 1978 & 0 & 4 \\
\hline 1979 & 0 & 2 & 1979 & 0 & 4 \\
\hline 1980 & 0 & 2 & 1980 & 2 & 6 \\
\hline 1981 & 0 & 2 & 1981 & 1 & 7 \\
\hline 1982 & 0 & 2 & 1982 & 2 & 9 \\
\hline 1983 & 0 & 2 & 1983 & 4 & 13 \\
\hline 1984 & 0 & 2 & 1984 & 3 & 16 \\
\hline 1985 & 1 & 3 & 1985 & 2 & 18 \\
\hline 1986 & 0 & 3 & 1986 & 2 & 20 \\
\hline 1987 & 0 & 3 & 1987 & 3 & 23 \\
\hline 1988 & 0 & 3 & 1988 & 0 & 23 \\
\hline 1989 & 0 & 3 & 1989 & 3 & 26 \\
\hline 1990 & 1 & 4 & 1990 & 2 & 28 \\
\hline 1991 & 0 & 4 & 1991 & 7 & 35 \\
\hline 1992 & 0 & 4 & 1992 & 2 & 37 \\
\hline 1993 & 1 & 5 & 1993 & 3 & 40 \\
\hline 1994 & 0 & 5 & 1994 & 3 & 43 \\
\hline 1995 & 1 & 6 & 1995 & 1 & 44 \\
\hline 1996 & 0 & 6 & 1996 & 2 & 46 \\
\hline 1997 & 2 & 8 & 1997 & 0 & 46 \\
\hline 1998 & 3 & 11 & 1998 & 4 & 50 \\
\hline 1999 & 0 & 11 & 1999 & 3 & 53 \\
\hline 2000 & 0 & 11 & 2000 & 1 & 54 \\
\hline 2001 & 0 & 11 & 2001 & 1 & 55 \\
\hline 2002 & 1 & 12 & 2002 & 1 & 56 \\
\hline 2003 & 2 & 14 & 2003 & 3 & 59 \\
\hline 2004 & 2 & 16 & 2004 & 2 & 61 \\
\hline 2005 & 1 & 17 & 2005 & 2 & 63 \\
\hline 2006 & 2 & 19 & 2006 & 0 & 63 \\
\hline 2007 & 8 & 27 & 2007 & 1 & 64 \\
\hline 2008 & 7 & 34 & 2008 & 3 & 67 \\
\hline 2009 & 5 & 39 & 2009 & 0 & 67 \\
\hline 2010 & 8 & 47 & 2010 & 1 & 68 \\
\hline 2011 & 4 & 51 & 2011 & 1 & 69 \\
\hline 2012 & 7 & 58 & 2012 & 1 & 70 \\
\hline 2013 & 8 & 66 & 2013 & 0 & 70 \\
\hline 2014 & 14 & 80 & 2014 & 1 & 71 \\
\hline 2015 & 11 & 91 & 2015 & 1 & 72 \\
\hline 2016 & 12 & 103 & 2016 & 1 & 73 \\
\hline 2017 & 1 & 104 & & & \\
\hline
\end{tabular}




\section{Artículos}

Después de tener la serie de datos para artículos, se aplicaron los trece modelos del software Sigmaplot, en la tabla 5, se observa los resultados para el parámetro de desempeño en artículos.

Tabla 5: Resultados de la aplicación de los trece modelos en artículos (Software Sigmaplot)

\begin{tabular}{|c|c|c|c|c|c|c|c|c|c|c|}
\hline Artículos & \multicolumn{10}{|c|}{ Harina de Carnaza } \\
\hline Validez & Modelo & $\begin{array}{l}\text { Punto de } \\
\text { inflexión }\end{array}$ & $\begin{array}{c}\mathrm{R} 2 \\
\text { Ajustado }\end{array}$ & Valor t & Valor t(a) & Valor t(b) & $\begin{array}{l}\text { Valor } \\
\mathrm{t}(\mathrm{Yo})\end{array}$ & Valor $\mathrm{p}$ & $\begin{array}{l}\text { Valor } \\
\mathrm{p}(\mathrm{Yo})\end{array}$ & $D-W$ \\
\hline $\mathrm{Si}$ & Sigmoidal 3 & 2021 & 0.9931 & 765.8524 & 3.6074 & 17.3938 & & $<0,0001$ & & 0.8053 \\
\hline $\mathrm{Si}$ & Sigmoidal 4 & 2016 & 0.9954 & 1733.401 & 7.9106 & 17.9133 & 5.3085 & $<0,0001$ & $<0,0001$ & 1.0569 \\
\hline No & Sigmoidal 5 & \multicolumn{9}{|c|}{ No cumple parámetros } \\
\hline $\mathrm{Si}$ & Logístico 3 & 2021 & 0.9931 & 739.7721 & 3.4967 & -17.2772 & & $<0,0001$ & & 0.8022 \\
\hline $\mathrm{Si}$ & Logístico 4 & 2016 & 0.9954 & 1702.272 & 7.7905 & -17.845 & 5.3259 & $<0,0001$ & $<0,0001$ & 1.0545 \\
\hline No & Weibull 4 & \multicolumn{9}{|c|}{ No cumple parámetros } \\
\hline No & Weibull 5 & \multicolumn{9}{|c|}{ No cumple parámetros } \\
\hline No & Gompertz 3 & \multicolumn{9}{|c|}{ No cumple parámetros } \\
\hline No & Gompertz 4 & \multicolumn{9}{|c|}{ No cumple parámetros } \\
\hline No & Hill 3 & \multicolumn{9}{|c|}{ No cumple parámetros } \\
\hline No & Hill 4 & \multicolumn{9}{|c|}{ No cumple parámetros } \\
\hline No & Chapman 3 & \multicolumn{9}{|c|}{ No cumple parámetros } \\
\hline No & Chapman 4 & \multicolumn{9}{|c|}{ No cumple parámetros } \\
\hline
\end{tabular}

Después de aplicar los trece modelos en la tecnología "Harina de Carnaza", se puede observar que de los trece modelos, Sigmoidal 3 y 4 parámetros y Logístico 3 y 4 parámetros, se ajustan a los parámetros estadísticos establecidos, entre los años 2016 - 2021, para esta tecnología, basados en el parámetro de desempeño de artículos y según el ciclo de vida, se encuentra en las fases de entrante y clave. (Figura 10)

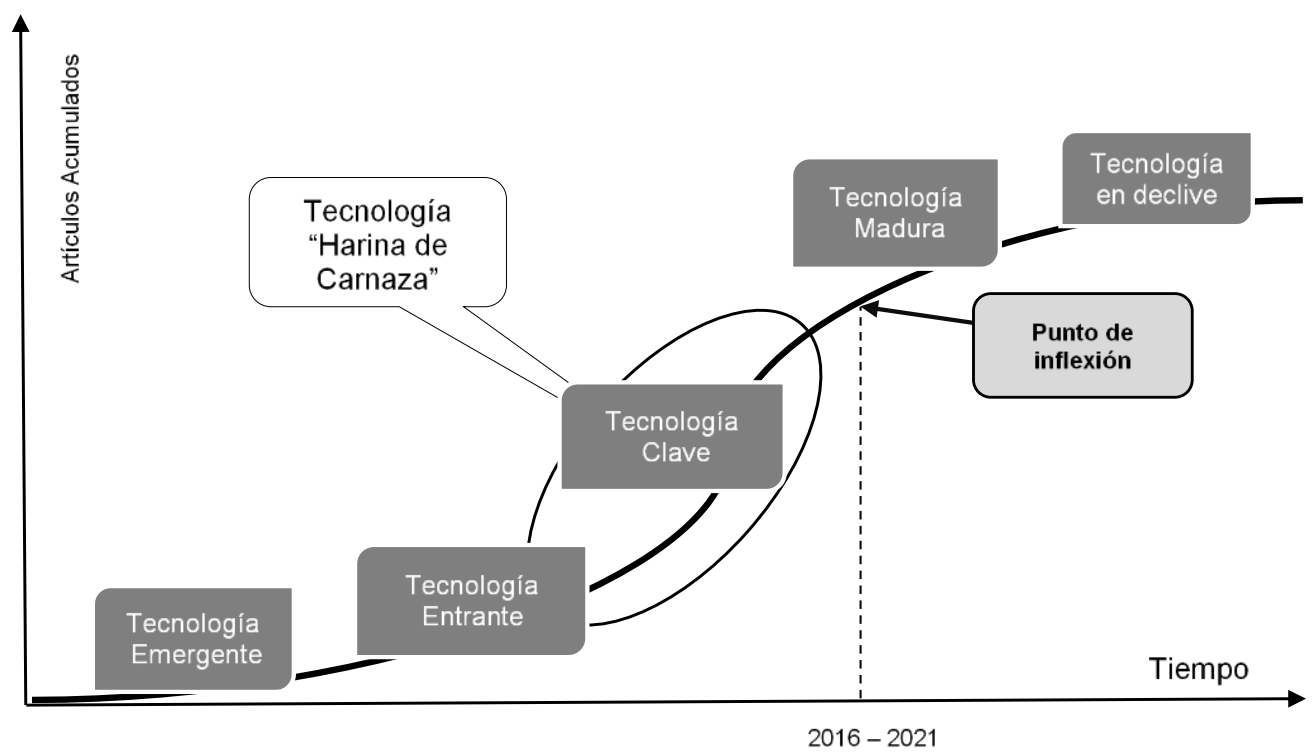

Fig. 10: Estado de la tecnología en artículos, adaptado de Ortiz y Pedroza (2006), citado por Zartha et al. (2015)

De acuerdo con los resultados y en cuanto a la identificación del estado de la tecnología (antes y después del punto de inflexión): el punto de inflexión entre los años 2016 - 2021, indica que la tecnología "Harina de Carnaza", desde el punto de vista de artículos, es una tecnología que está en transición de entrante a clave. (Figura 10), por lo que se presenta una oportunidad para los académicos e investigadores de publicar en esta 
área de interés tecnológica. Por otra parte, la determinación del momento adecuado para aplicar mecanismos de derecho tecnológico y propiedad intelectual: el punto de inflexión entre los años 2016 - 2021, indica que la tecnología "Harina de Carnaza", está teniendo un crecimiento rápido a crecimiento tardío, por lo que puede ser una tecnología de poco conocimiento a ser de conocimiento accesible. (Figura 11)

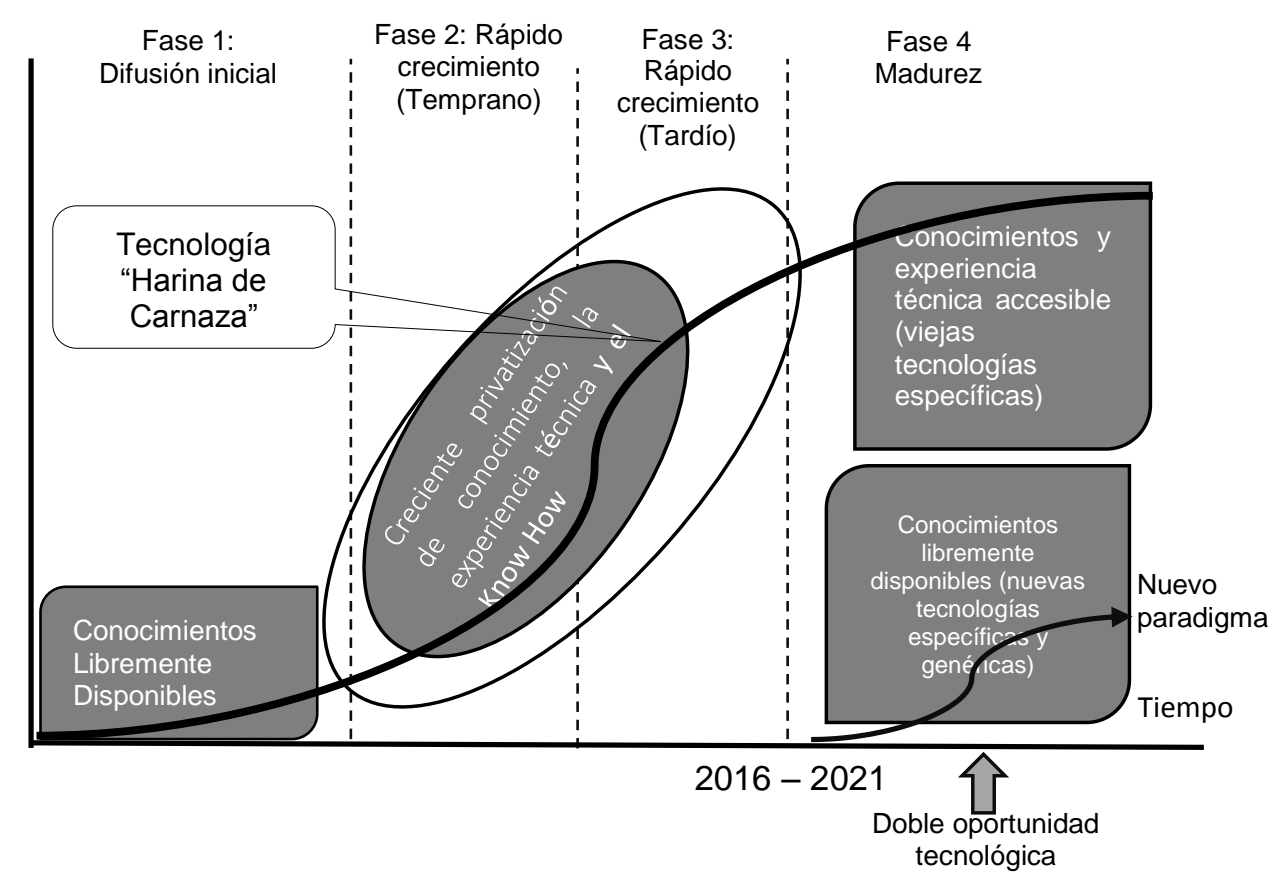

Fig.11: Mecanismos de derecho tecnológico y propiedad intelectual en artículos, adaptado de Pérez $(1988,2001)$ citado por Zartha et al. (2014)

Establecimiento de estrategias adecuadas de monitoreo e inversión: como el punto de inflexión está entre los años 2016 - 2021, indica que la tecnología "Harina de Carnaza", se encuentra en fase de transición de tecnología de punta a clave, es conveniente invertir selectivamente y tratar de construir con aliados estratégicos, a su vez, se puede realizar publicaciones conjuntas con centros o investigadores de otros países. (Figura 12)

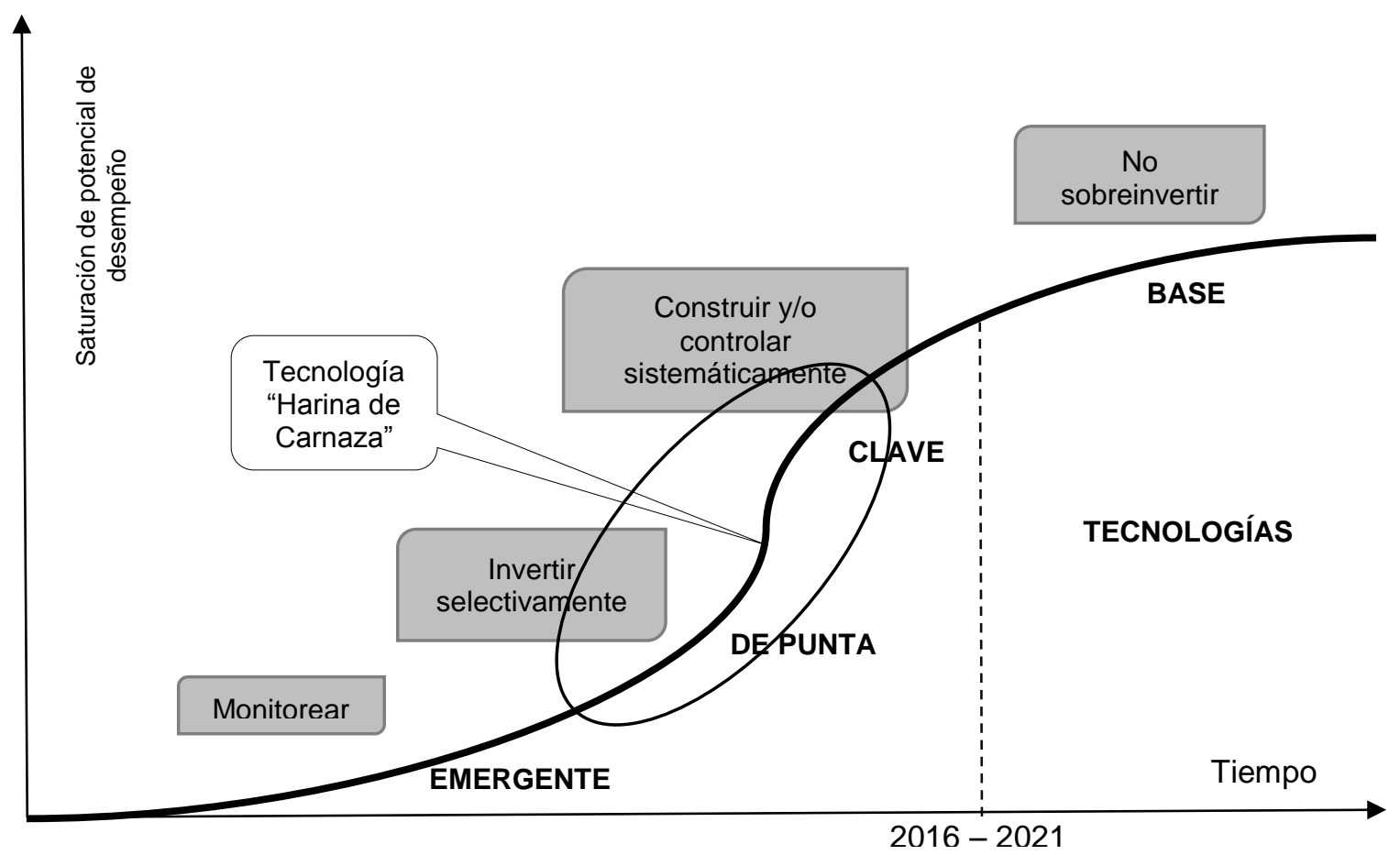

Fig.12: Estrategias adecuadas de monitoreo e inversión en artículos, adaptado de Ortiz y Pedroza (2006) 


\section{Patentes}

Por último, en cuanto a la serie de datos para patentes, se aplicaron los trece modelos del software Sigmaplot, en la tabla 6, se observa los resultados para el parámetro de desempeño en patentes.

Tabla 6: Resultados de la aplicación de los trece modelos en patentes (Software Sigmaplot)

\begin{tabular}{|c|c|c|c|c|c|c|c|c|c|c|}
\hline Patentes & \multicolumn{10}{|c|}{ Harina de Carnaza } \\
\hline Validez & Modelo & \begin{tabular}{|c|} 
Punto \\
de \\
inflexión
\end{tabular} & $\begin{array}{c}\mathrm{R}^{2} \\
\text { Ajustado }\end{array}$ & Valor t & $\begin{array}{l}\text { Valor } \\
\text { t(a) }\end{array}$ & $\begin{array}{l}\text { Valor } \\
\mathrm{t}(\mathrm{b})\end{array}$ & $\begin{array}{l}\text { Valor } \\
\mathrm{t}(\text { Yo })\end{array}$ & Valor $\mathrm{p}$ & $\begin{array}{l}\text { Valor } \\
\mathrm{p}\left(\mathrm{Yo}_{\mathrm{o}}\right)\end{array}$ & D-W \\
\hline $\mathrm{Si}$ & Sigmoidal 3 & 1992 & 0.9953 & 7808.687 & 86.8312 & 31.9111 & & $<0,0001$ & & 0.4993 \\
\hline $\mathrm{Si}$ & Sigmoidal 4 & 1991 & 0.9966 & 6831.621 & 39.2092 & 21.185 & -3.5498 & $<0,0001$ & 0.001 & 0.7009 \\
\hline No & Sigmoidal 5 & \multicolumn{9}{|c|}{ No cumple parámetros } \\
\hline $\mathrm{Si}$ & Logistico 3 & 1992 & 0.9953 & 7822.432 & 86.8259 & $\begin{array}{c}- \\
32.0792\end{array}$ & & $<0,0001$ & & 0.506 \\
\hline Si & Logistico 4 & 1991 & 0.9966 & 6878.283 & 39.4016 & $21 . \overline{2623}$ & -3.5133 & $<0,0001$ & 0.0011 & 0.705 \\
\hline Si & Weibull 4 & 1992 & 0.9972 & 6933.803 & 55.1251 & 18.7334 & & $<0,0001$ & & 0.8261 \\
\hline $\mathrm{Si}$ & Weibull 5 & 1993 & 0.998 & 4678.744 & 39.6385 & 31.8183 & 6.5094 & $<0,0001$ & $<0,0001$ & 1.1788 \\
\hline $\mathrm{Si}$ & Gompertz 3 & 1989 & 0.9978 & 10018.2 & 80.627 & 36.658 & & $<0,0001$ & & 1.0013 \\
\hline No & Gompertz 4 & \multicolumn{9}{|c|}{ No cumple parámetros } \\
\hline No & Hill 3 & \multicolumn{9}{|c|}{ No cumple parámetros } \\
\hline No & Hill 4 & \multicolumn{9}{|c|}{ No cumple parámetros } \\
\hline No & Chapman 3 & \multicolumn{9}{|c|}{ No cumple parámetros } \\
\hline No & Chapman 4 & \multicolumn{9}{|c|}{ No cumple parámetros } \\
\hline
\end{tabular}

Después de aplicar los trece modelos en la tecnología "Harina de Carnaza", se pudo observar que de los trece modelos, Sigmoidal 3 y 4 parámetros, Logístico 3 y 4 parámetros, Weibull 3 y 4 parámetros y Gompertz 3 parámetros se ajustan a los parámetros estadísticos establecidos, entre los años 1989 - 1993, esta tecnología, basados en el parámetro de desempeño de patentes y según el ciclo de vida, se encuentra en la fase de tecnología madura. (Figura 13)

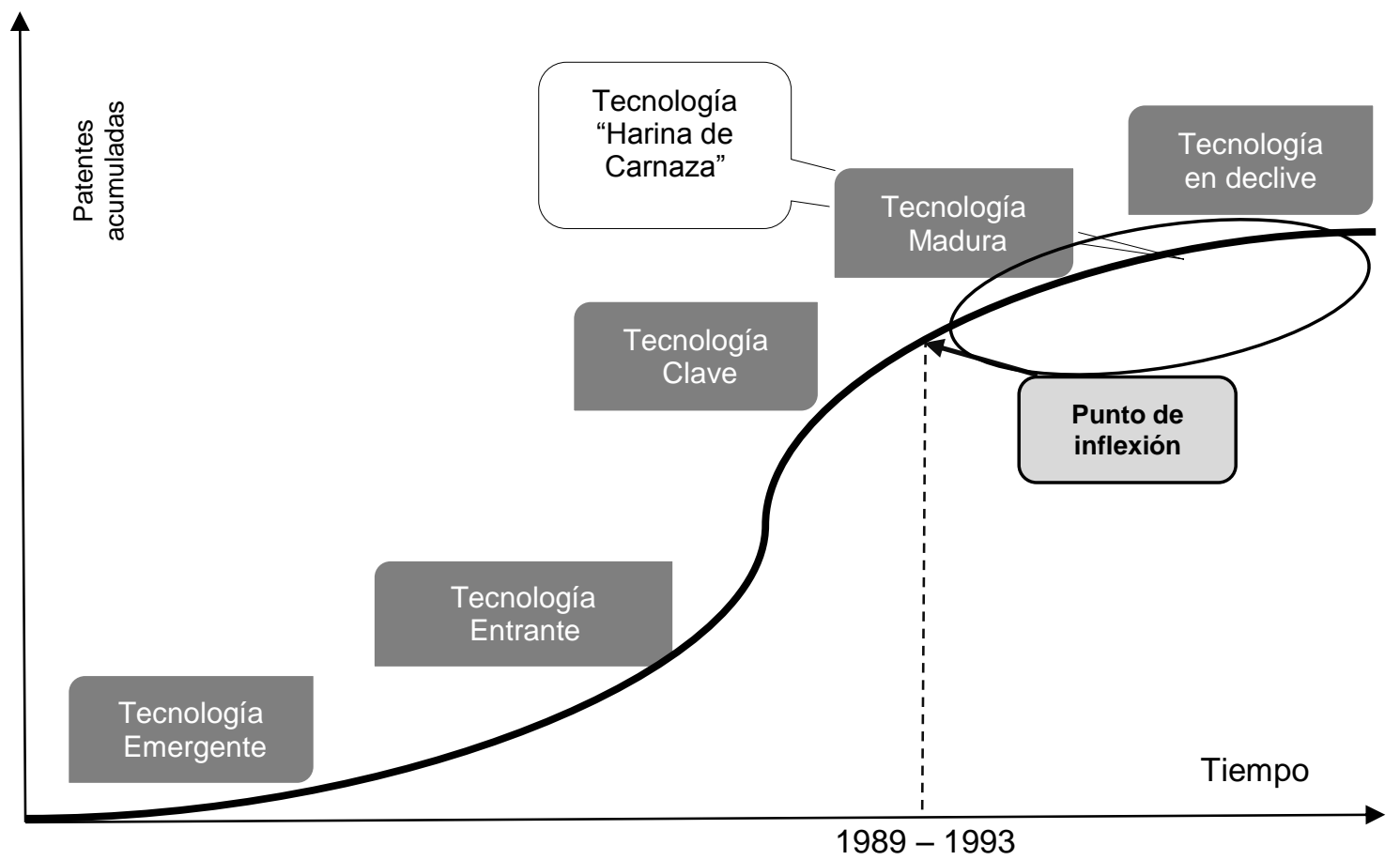

Fig. 13: Estado de la tecnología en patentes, adaptado de Ortiz y Pedroza (2006), citado por Zartha et al (2014)

De acuerdo con los resultados y en cuanto a la identificación del estado de la tecnología (antes y después del punto de inflexión): el punto de inflexión entre los años 1989 - 1993, indica que la tecnología "Harina de 
Carnaza", desde el punto de vista de patentes, es una tecnología que está en fase madura. (Figura 13), por lo que se recomienda buscar otro tipo de áreas de interés en las cuales se pueda llegar a proteger o comprar tecnología para el procesamiento de carnaza. Por otro lado, la determinación del momento adecuado para aplicar mecanismos de derecho tecnológico y propiedad intelectual: el punto de inflexión entre los años 1989 - 1993, indica que la tecnología "Harina de Carnaza", está en una fase de madurez, por lo que es una tecnología de conocimiento y experiencia técnica accesible. (Figura 14)

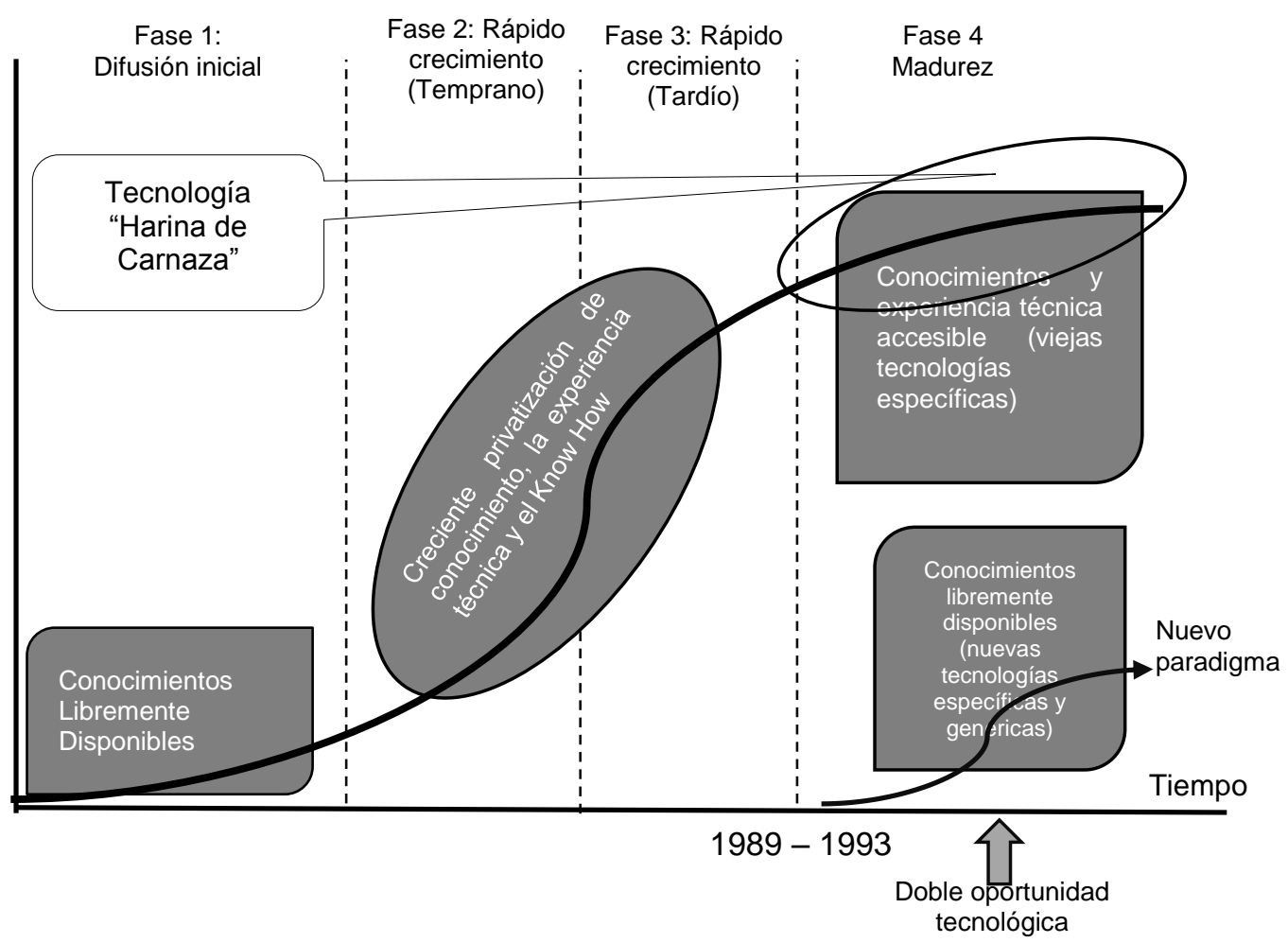

Fig.14: Mecanismos de derecho tecnológico y propiedad intelectual en patentes, adaptado de Pérez $(1988,2001)$ citado por Zartha et al. (2014)

Establecimiento de estrategias adecuadas de monitoreo e inversión: como el punto de inflexión está entre los años 1989 - 1993, indica que la tecnología "Harina de Carnaza", se encuentra en fase de no sobre invertir, por lo que es conveniente tratar de construir con las capacidades actuales o co desarrollar con centros o investigadores de otras empresas interesadas en este proceso. (Figura 15)

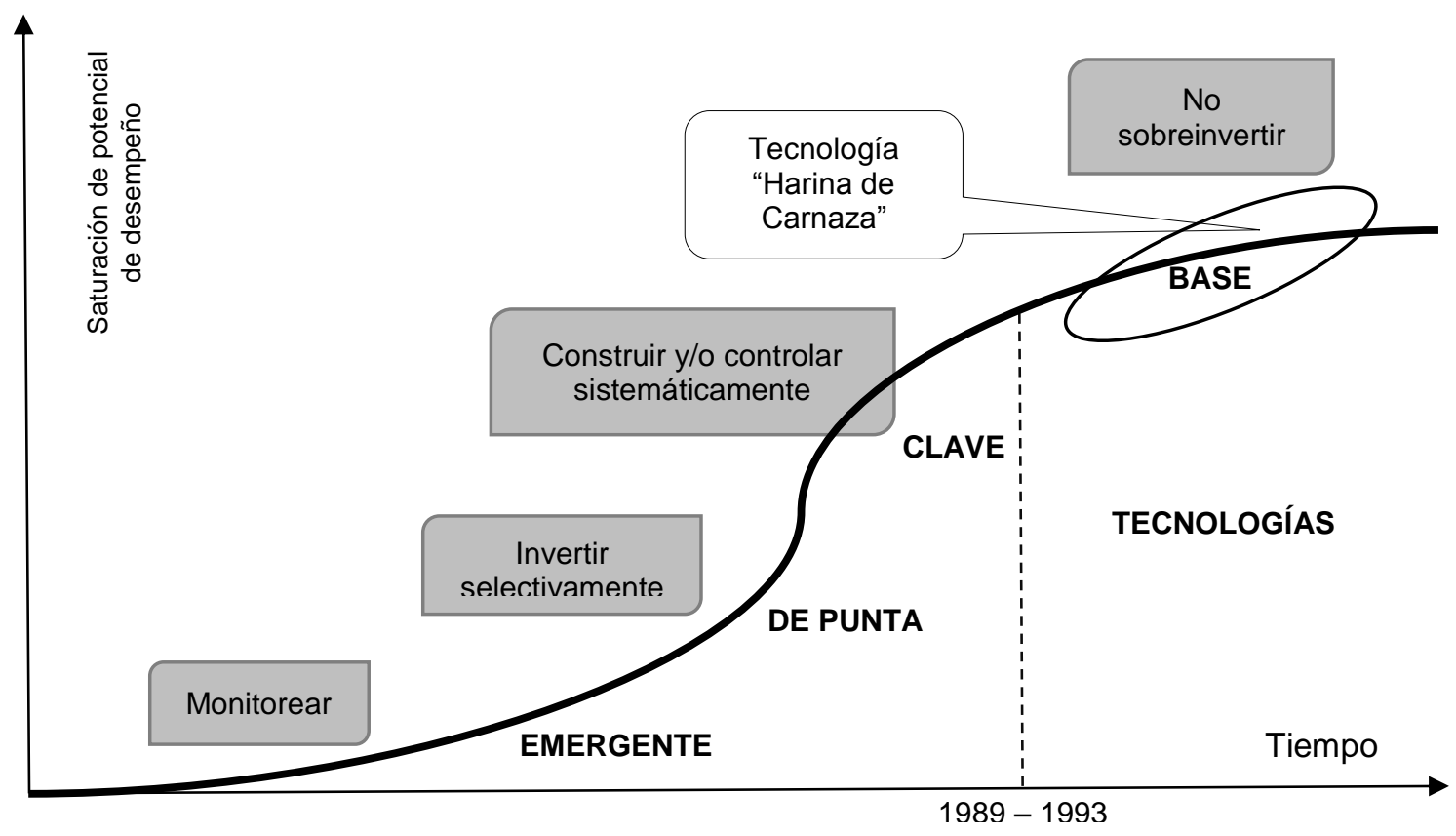

Fig. 15: Estrategias adecuadas de monitoreo e inversión en patentes, adaptado de Ortiz y Pedroza, (2006) 
Fase de comunicación: en esta fase, se realizó un infográfico que tuvo como finalidad recopilar la información obtenida en cada una de las fases anteriores de la vigilancia tecnológica y de curvas en S, esto con el fin de socializar la información a José Bestier Padilla Docente Investigador de la Universidad del Quindío y José Joaquín Duque Coordinador general de la asociación de curtidores de la María.

\section{CONCLUSIONES}

La Vigilancia Tecnológica, permitió conocer la dinámica en cuanto a publicaciones, y en especial de instituciones que están liderando las investigaciones en el tema principal de este estudio, esto en cuanto a artículos; en patentes se observa los países en los cuales más se protege por medio de patente en el tema trabajado, y su comportamiento en el tiempo en cuanto a las solicitudes.

Los países con mayor publicación de artículos fueron Estados Unidos, China, Reino Unido, Canadá, Hong Kong y Taiwán, mientras que las instituciones con mayores aportes sobre derivados de la curtición en el periodo de tiempo analizado fueron Pennsylvania State University, Hong Kong University of Science and Technology, Chalmers University of Technology", Universidade Federal do Rio Grande do Sul, National Chiao Tung University Taiwan, University of Windsor", Georgia Institute of Technology, Sultan Qaboos University, The George W. Woodruff School of Mechanical Engineering" y Siksha O Anusandhan University.

En cuanto nuevos productos y nuevas aplicaciones de la carnaza se encuentran las láminas de poliuretano adheridos a cuero, revestimiento de textiles con poliuretanos, aplicaciones para el sector automotriz para para asientos de automóviles, aviones, motos, usos como guantes de cuero siliconado, cartucheras; faltriqueras, bolsas; zapatos de golf, cuero para cabrilla de automóviles, juguetes para aves, bolas de béisbol, películas de poliuretano micro porosa sobre cuero y colágeno para gelatina.

Uno de los resultados más importantes fue la descripción de dos nuevos procesos de elaboración para la fabricación de juguetes caninos que involucran operaciones como, evaporación, laminación, calentamiento dieléctrico, gofrado, prensado térmico, proceso de preparación, proceso de curtido, proceso de pre-teñido, proceso de teñido, proceso de secado, proceso de acabado, proceso de bronceado, proceso de punzonado, textiles tricotados multicapa, revestimiento de fondo, recurtido, proceso de encalado, estos procesos se describen en dos patentes de Estados Unidos.

En cuanto al punto de inflexión obtenido en patentes 1989 - 1993 se puede concluir que, bajo este parámetro de desempeño, la tecnología se encuentra en fase de madurez o declive, es decir, es una tecnología de base, e indica que la dinámica de patentamiento sobre ésta tecnología ha disminuido desde ese periodo de tiempo.

Desde el punto de vista de los artículos, el punto de inflexión generó un valor en el rango 2016 - 2021 lo que demuestra que aún existe una alta dinámica en publicaciones en Scopus y representa una oportunidad para los académicos e investigadores en continuar investigando y publicando en esta tecnología.

\section{REFERENCIAS}

Aenor. Asociación española de normalización y certificación. Gestión de la l+D+i. Requisitos del sistema de gestión de la I+D+i, Pub. L. No. UNE 166002 España (2006)

Burgelman, R., C. Christensen y S. Wheelwright, Strategic Management of technology and innovation, 5a Edición, McGraw-Hill, Boston (2009)

Cámara de Comercio de Bogotá, Guía Ambiental Para El Sector Curtiembres (2004)

Centro Nacional de Producción Más Limpia, Diagnóstico ambiental del sector curtiembre en Colombia (2004)

Dosi, G., Technological paradigms and technological trajectories: A suggested interpretation of determinants and directions of technical change, Research Policy, 2(3) (1982)

Foster, R., Working the S-Curve: Assessing Technological Threats, Research Management, 29(4) (1986)

Freeman, P. y C. Lemen, Puncture-Resistance of Gloves for Handling Bats. The Journal of Wildlife Management, 73(7) (2009)

Konrad, C., K. Lorber y otros siete autores, Systematic analysis of material fluxes at tanneries, Journal of the Society of Leather Technologists and Chemists, 86 (1), 18 - 25 (2002)

Kucharavy, D. y R. De Guio, Application of S-Shaped Curves. TRIZ-Future conference 2007, Current Scientific and Industrial Reality, Frankfurt (2007)

$\mathrm{Li}$, N., K. Chen y M. Kou, Technology Foresight in China: Academic studies, governmental practices and policy applications. Technological Forescasting and Social Change, 119, 246-255 (2017) 
Manderieux, L., Guía práctica para la creación y la gestión de oficinas de transferencia de tecnología en universidades y centros de investigación de América Latina, El rol de la propiedad intelectual, Organización Mundial de la Propiedad Intelectual (2011)

Milašienè, D., Effect of environment temperature on fatigue properties of laminated leather, MECHANIKA, 6(68) (2007)

Nelson, R. y S. Winter, In search of a useful theory of innovation, Research Policy, 6(1), 36-76 (1977)

Ortiz, S. y A. Pedroza, ¿Qué es la gestión de la innovación y la tecnología (GInnT)? Journal of Technology Management and Innovation, 1 (2) (2006)

Pérez, C., Cambio tecnológico y oportunidades del desarrollo como blanco móvil, Revista de la Cepal, 75, 115-136 (2001)

Sánchez, J. y F. Palop, Herramientas de software para la práctica en la empresa de la vigilancia tecnológica e inteligencia competitiva, Evaluación comparativa, Valencia, España, Triz XXI (2002)

Shilling, M., Strategic management of technological innovation, 4ª Edición, McGraw-Hill, New York (2013)

Schilling, M. y M. Esmundo, Technology S-curves in renewable energy alternatives: Analysis and implications for industry and government, Energy Policy, 37(5,) 1767-1781 (2009)

Schmelzer, H., Polyurethanes for Flexible Surface Coatings and Adhesives, Journal of Industrial Textiles, 17(3), 167 182 (2016)

Tidd, J. y J. Bessant, Managing Innovation, Integrating Technological Market and Organizational Change, 5ª Edición, Reino Unido (2013)

UNE 166006, Gestión de la I+D+i: sistema de vigilancia tecnológica e inteligencia competitiva (2011)

Zartha, J., F. Palop y otros tres autores, S-Curve Analysis and Technology Life Cycle, Application in series of data of articles and Patents, $3^{\text {rd }}$ Global TechMining Conference, Atlanta, Georgia (2013)

Zartha, J., H. Villada y otros nueve autores, Application of Technological Intelligence Tools and S-Curves in a Foresigth Evaluation Regarding Biodegradables Packaging and Environmentally Friendly Up to 2032, Revista Espacios, ISSN: 0798-1015, $36(9), 18-19(2015)$ 
